\title{
(Ei $\mathfrak{i} \mathfrak{l} \mathfrak{e} \mathfrak{i} \mathfrak{t} \mathfrak{n} \mathfrak{g}$.
}

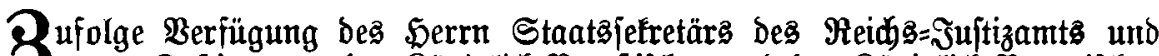

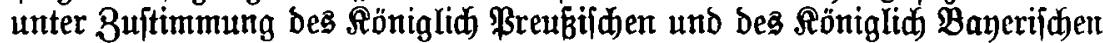

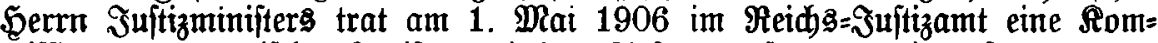
mifjion von praftijळen Эuriften mit bem $\mathfrak{A}$ uftrag zufammen, einen formulierten Borentwurf zu einem neuell Deutiden Strafgejebbud nebit Begrünoung auzzuarbeiter. Der Sommijijion gebörten an: $\left.{ }^{1}\right)$

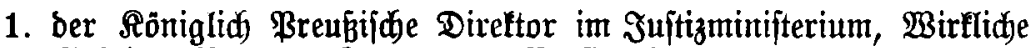
Beheime Rat Dr. Bucas als Borjigenber,

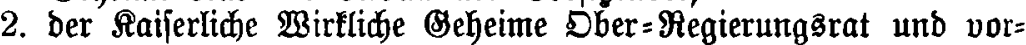

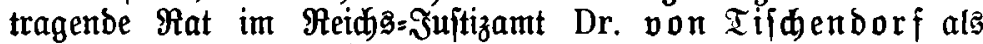
Stellwertreter bes Borjizenden,

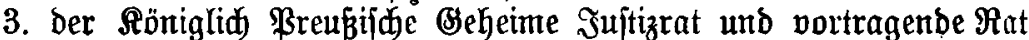
im $\mathfrak{J} \mathfrak{u} \mid \mathrm{t} \mathfrak{z}$ minifterium Dr. $\mathfrak{S}_{\mathfrak{j}} \mathfrak{u} \mathfrak{l}$,

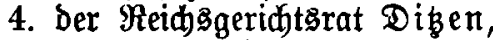

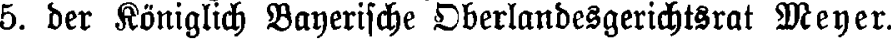

Da fid Geheimrat Dr. von Tifdendorf feit Dem Gerbjt 1907

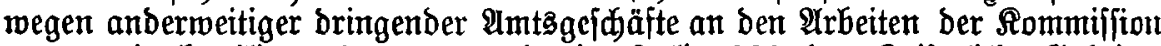
nur wenig beteiligen Eonnte, wurbe im Jult 1908 ber Raijerlidje Beheime

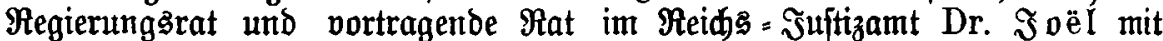
jeiner $\mathfrak{B e r t r e t u n g ~ b e a u f t r a g t . ~ F e r n e r ~ w a r ~ f e i t ~ b e m ~ S e r b f t ~} 1908$ Gebeimrat

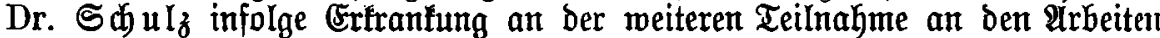
Der Rommiffion verfindert; an jeine Stelle trat Der Rontiglid ßreutifide

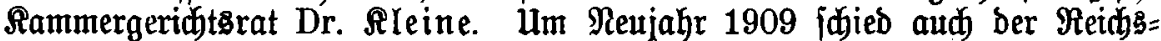

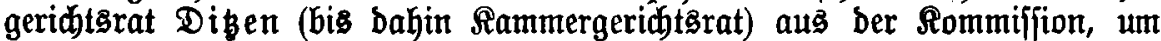

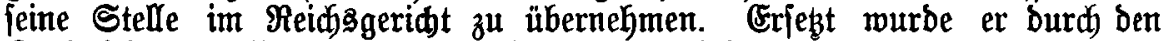

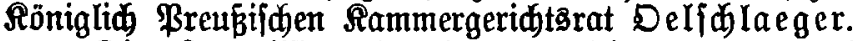

Die Rommiffion hat in 117 Sibungen, von benen bie lebte am 22. 2)pril 1909 ftattfanb, ifhe Uufgabe erlebigt unb legt nummehr ben fertigen Entwurf nor. Diejer ift fein amtlider, fonbern enthält lebiglid bie

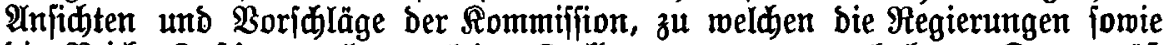

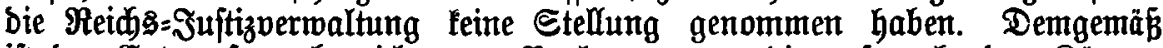
ijt ber Entwurf aud nidjt zur Borlegung an bie gejebgebenden Rörper=

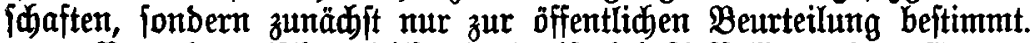

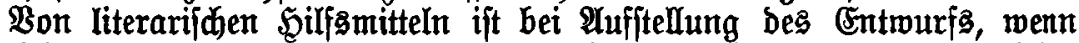

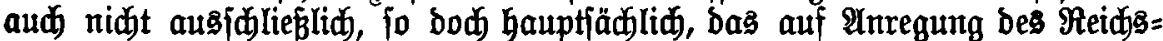

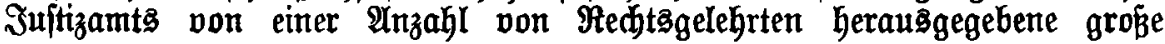

1) Bei einigen Der Mitglieder haben im Berlaū ber Beratungen die Tmtstitel ñ̆f geändert. Şier find die gegenwärtigen aufgejührt. 
Sammelmerf "Berglcidende Daritellung Des Deutiden und ausländijdlen

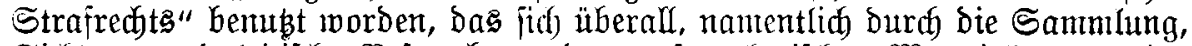
Sidfung und fritifbe Beiprechung Des gefebgeberijben Materials, als eine jefr wertodle Brundlage crwiejen hat. Daburd, ift aud bie Beigabe von

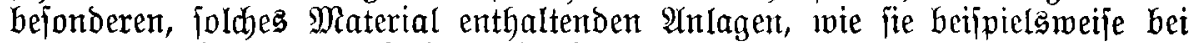

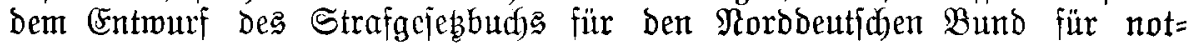
wenbig gehalten wurbe, überflüfigg geworden, vielmehr fann in biejer Sinjidt überall auf bie "Bergleichende Darjtelfung" verwiejen merben. Die in biejer

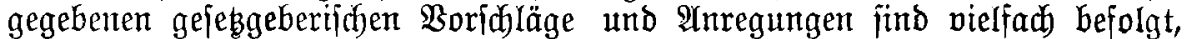
immer aber in ihren wejentlidjten ßunften beadytet und erörtert worben. ¿̈fters ift pon ibnen audh abgewidhen, da die Sommiffion auf eine freie, auf praftifdhe (Erfahrungen grgründete Beurtelung umpomeniger verziddten

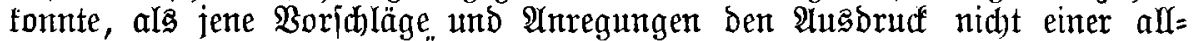
gemeinen wiffenjhaftlidben überzeugung, fondern nur Derjentigen bes einzelnen $\mathfrak{B}$ earbeiters enthalten. Hber aud mo ignen nicht beigepflidftet werden tonnte, füb fie Der $\mathfrak{A}$ rbeit Der Rommiffion förberlidf geweien.

Bas ben Jnhalt Des Entwurfs anbelangt, jo ift zunädfft zmei in ber

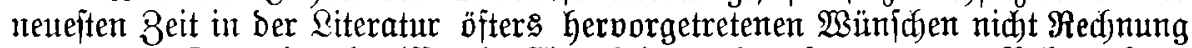
getragen. Der eine betrifft dic Cimarbeitung Der fogenamten Nebengejebe,

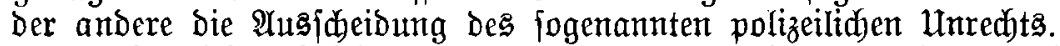

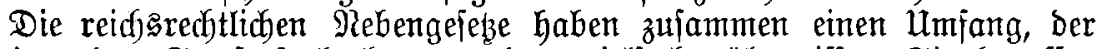
denjenigen des Strafgefesbudis um das vielfadie überirifft. Sie betrefien meijt an fid nidgt frafredbtlidge Materien, benen nur gewiffe Straf $=$ beftimmungen angefbloffen find. Injomeit eignen jie find, wie z. B. Die

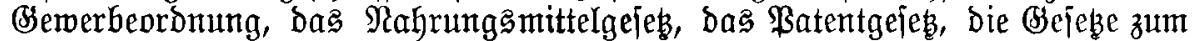

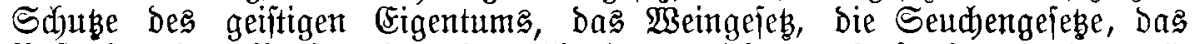
Pojtgejes, das Börjengejeb uiw., überfaupt nidgt zur aufnahme in bas all= gemeine Strafgejebbuch. Diejes mürbe baburch nidbt nur einen ganz über= ntäbigen Ulmfang erhalten, jonbern aud mit einer Menge frembartiger Materien angefülut werben und Den Ramen eines Strafgefesbud) überhaupt nidft mehr verdienen. Daher ifjt in joldher Weije aud noch feime andere Sation verfahren, fondern überall haben die Strafgejeşbüber annähernd etra Denfelben Bereidy der barin behandelten Gegenftände, wie das Deutfde, zum Teil einen geringeren. Das Strafgejebbuh ijt nirgenos eine Sammlung aller beftehenden ftrafgejebslicben Borjdyriften, fonbern enthält überall unr

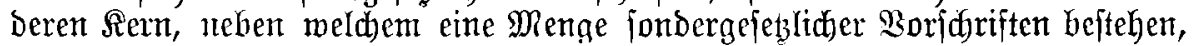
bie mit befonderen Bebürinifijen pon Staat unb Gejcllidaft zujammenhängen und nidht in bemielben Sinne gemeines Strafredt jumb. Sonnte es jidy Danad) hödhftens Darum hanbeln, einige wenige Rebengejebe rein ftrafredt= richer Ratur bem Entwurf einzunerleiben, jo war aud hierin Borjidyt ge=

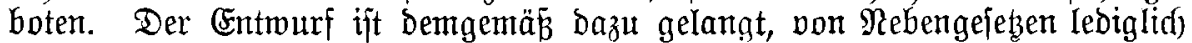

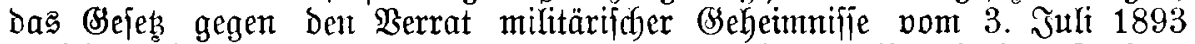

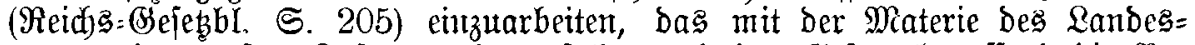
verrats im engiten Bujammentang fteht, und Das Bejes, betreffend die $\mathfrak{B e}=$ ftrafung Der (Entziehung eleftrîd)er Srbeit, vom 9. April 1900 (Reid) S. 228) Durd feine Beftimmungen überflüfitg zut macten. Dagegen find

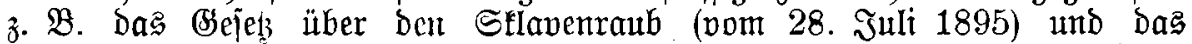
Sprengftoffgeieb (pom 9. Эuni 1884) zur Einarbeitung nidbt für geeignet

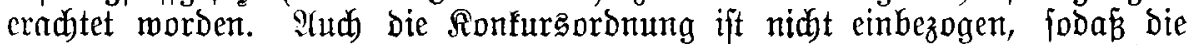
Beftimmungen über Den Banferott auberhalb Des Strafgejebbuds Gleiben. Dar in biejes bie Ronfursorbnung als Banzes nicht gehört, fanm nicht 
zmeifelhaft fein. Sie Duraf Übernahme ber gegen Den Banferott geridteten Beftimmungen zu zerreipen, eridjien aber nidjt angemejen, zumal dieje crij vor furzem revibiert worden find und einer Seubronung zur Beit nitift be= Dïrịen.

Der Entrourf enthält jidh aljo eines (singreifens in bie bejtebende Rebengejebgebung. Die Sidhtung und Robififation der Rebengeiebe mag eine 2ufgabe ber Bufunft jein, mit ber Shopping eines neuen Strafgejes= budjs hat fie bagegen nidht notwendig zu tum, im Segenteil mürbe jie bie

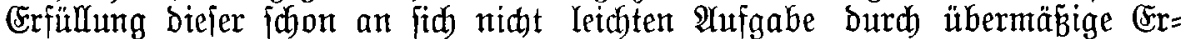

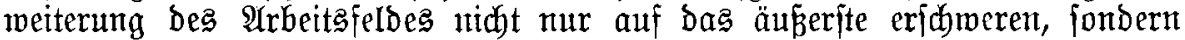
wahridjeinlid fogar vereiteln.

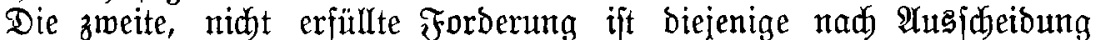

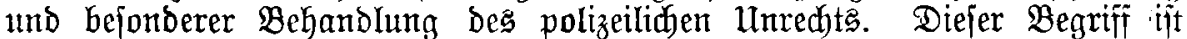
ein zmar theoretifd aufgeitellter, aber praftifd in feiner Weife fidher gefenn= zeidneter uno begrenzter. Shon die Motwe zu unjerem jebigen Stra neiebbuch jagen mit $\Re e(d):{ }^{1}$ )

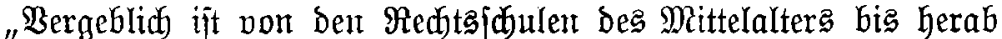
auf unjere Beit trobs ber Borliebe, welde man ber Frörterumg biejer Frage gemiomet hat, ber Berjud gemadyt worben, bie Grenzlinie zwifhen bem triminell und polizeilidg Strafbaren zu finden."

und ähnlid bemerft Seuffert:

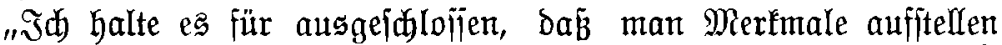
fann, bie gecignet find, in begrifflidyer alfgemeinfeit und mit praftijcher Durchführbarfeit bas polizeilidge und vermaltungsrecht= lidye Unredst vom friminellen, die Ulbertretung vont Bergeben (Ber= brechen) zu joiben. "2)

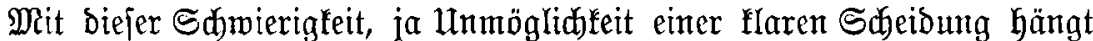
es zujammer, daßj es unter bon jebigen Bergehert, und zmar nidft mur im (Sebiet des Strafgejebbuds, jondern weit mehr nod) in Demjenigen ber $10=$

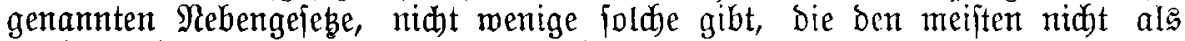
Red)ts̄verbredben, fondern als Polizeibelifte er/d)einen werben, andere, bei Denen bie Ilnterbringung pölig zweifelhaft unb nur wilfürlid zu voll= ziehen ijt, und mieberum übertretungen, die als Rechtsbeliłte angejeben werben müfien. Sn die erfte Rategorie gebören im Strafgejeb̧ud z. B. Die $\S \S 116,127,128,175,284$ bis 286, in bie lebteren beiben bie widtigen

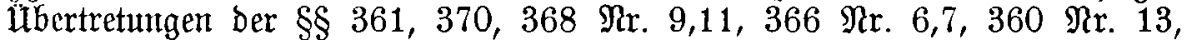

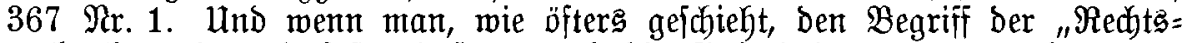

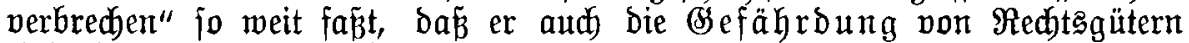
einid)ließst, jo wirft fid bie Frage auf, ob es bann überbaupt nod viele Strafporidyriften gibt, bie als polizeilidjes unredht angeieben werden fönnen. Denn bei weitem bie meiften biefer Borjdriften jind erlaffen, um ernjte Ge= fähroungen von $\mathfrak{Q}$ ben und (sigentum zu verhüten. So z. $\mathfrak{B}$. Die gegen Das unterlaffene Reinigen ber Sdornjteinte, anjheinend eine geringfügige Polizeiübertretung, bie aber zur Berhütung von Feuersgejahr unter Strafe

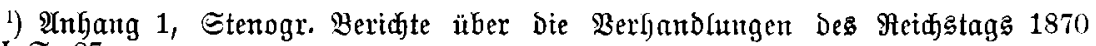
(3). III ङ. 87 .

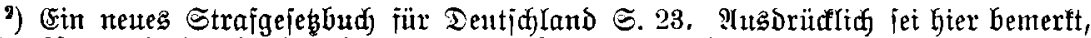

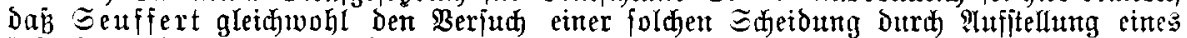
bejonderen Bolizetjtrafgejesbudse empfieglt. 
gejtellt ijt und für bie öfientlidbe Sidberheit cine groß̈e Bedeutung habert

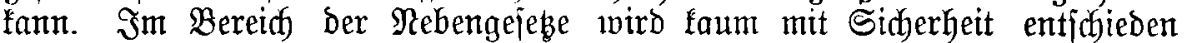
merben f̈̈nnen, ob z. $\mathfrak{B}$. Die Sorjhriften über dą firebitieren von $\mathfrak{B a r e n}$

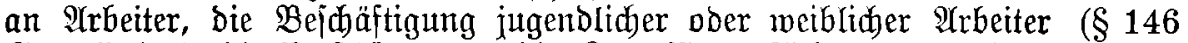

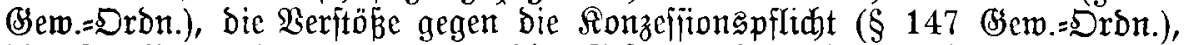
bie Buwiberhanblungen gegen bie Bejebe über bie Arbeiterverjidjerung,

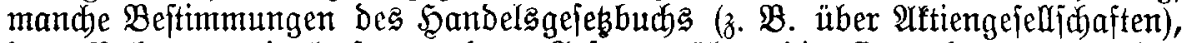
des Rahrungsmittelgejezes, Des Bsejebes über die Ermerbs= und Wirt=

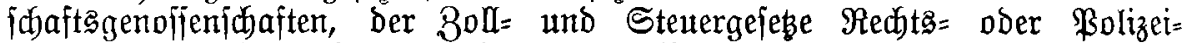
voridbriften find. Sbenjo wird man zmeifellaft jein müffen, ob z. B. Die

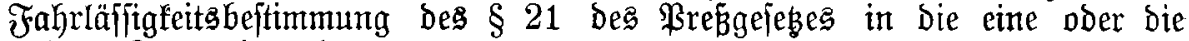
andere Rategorie gefört.

Sit hiernad bie angeregte Sheidung überhaupt praftijh unburdführ bar, fo wïrde jie jogar faum nod einen Sinn haben, wollte man jie

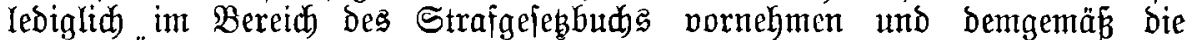

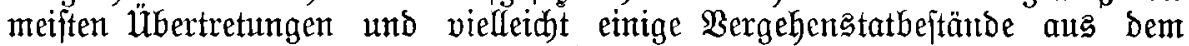

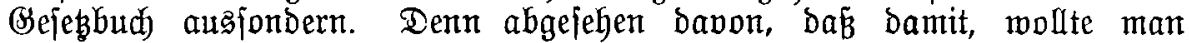

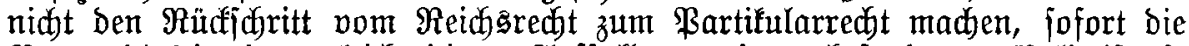

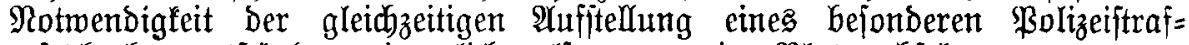

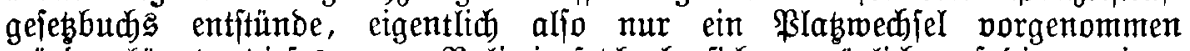
mürDe, fönnte Diejes neue ßolizeigejebsud fid unmöglid auf bie menigen

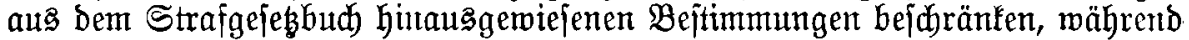
Daneben auf Dem ungeheuren (Gebiet Der Rebengejebe Die Bermifdyung pon jogenanntem polizeiliden und friminellem Unredht meiter bejtehen bliebe. Sollte aber aud auf Dem Gebiet Der Rebengeiebe zu ber Sidjung und

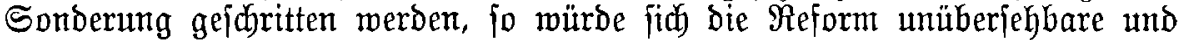
zur Beit nod faum durdhführbare Biele fteffen, die, abgejeben von ber cin=

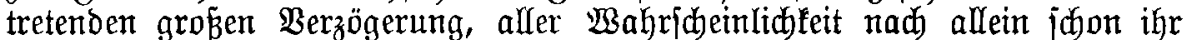
Sdyettern bedingen würben. Bei einer ernftlidy unternommenen und auf praftijhen (Erfolg angelegten Reform ijt bas erfte (Sebot, fich erreidbare Biele zu feben und weit ausjebende, zur Beit himjichtlich ihrer Röjung nod

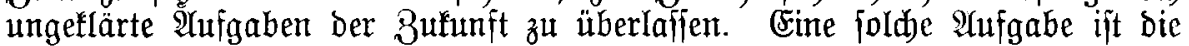
hier beiprodiene Schcibung, won ber man zmeifelm fant, ob fie jemals gelingen wird. Daher haben audh alle amberen modernen Gejebgebungen von ihr abgejeben. Die Nieberlande, Normegen, Stalien und bie Shmeiz haben bie Trennung nidst vorgenommen und Den lubertretungsabjdnitt im Straf = gejebbud nidht nur beibebalten, jonbern zum Teil fogar gegenüber Dem

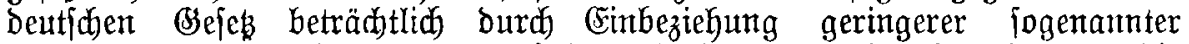
"Redtgdelifte" ermeitert. So verfährt, abgejehen pon Der Erweiterung, die er nidst vornimmt, aud ber vorliegende Entwuri. Das Berlangen nad

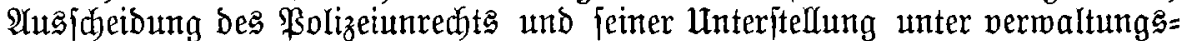

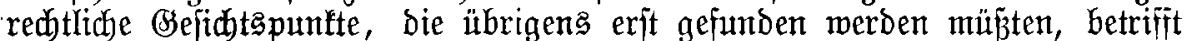

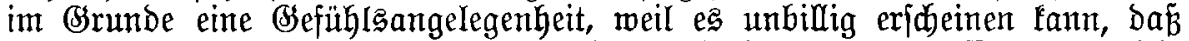
z. B. Derjenige, Der bie Inmeldung eines Dienftboten unterlajien ober nidht redftzeitig bewirft hat, mit einer Strafe belegt wirb, bie, mag fie audh mur eine geringe Beldfirafe jein, won frimineller Strafe nidjt jidtbar gejdieden ift. In Der Bevölferung fiebt aber obnehin niemand eine foldhe Bejtrafung als eine friminelle an. Wollte man Deffenungeadytet für joldhe Straffälle bejondere Regeln und Strafen jöaffen, Dann würbe man ihren Bereidh, ım unlößbaren Sdwierigkeiten und ïblen Ronjequenzen, namentliḑ auf bem Gebiet ber Rebengejese, zu entgehen, jebenfalls redt vorfichtig und eng 


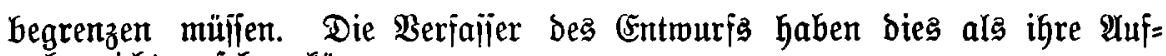
gabe nidjt amiehen fönner.

Sit jo ber Bereid) Des (Entwurfs im wejentliden auf Denjenigen bes gegentwärtigen Strafgejełbutbs beftimmt, fo erübrigen fidh jebt nod einige Borbenterlungen über feinen $\mathfrak{s}$ halt.

Rein Eimfichtiger wirb von ihm als Ganzem etwas vollifünoig Neues, nod nie Dagemejenes erwarten oder fordern fönnen. Die Straf =

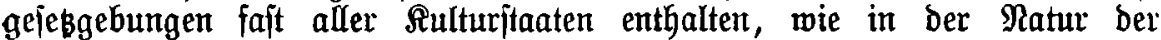

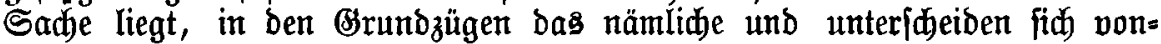
einanber mehr nur in allerbingg zahlreidjen (Finzelheiten. Ein Strafgefeb=

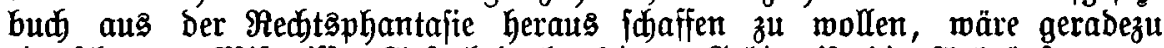

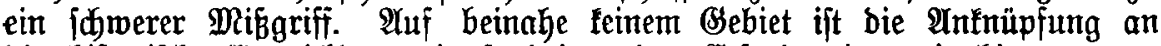
bie hiftorifale (Entwidłlung ein jo bringenbes Erforbernis, wie hier, wo es

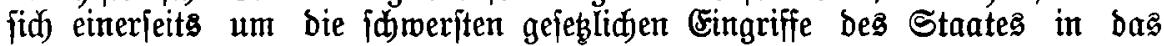
Qeben Der Bürger, anbererjeits um Den Sdub von Staat und Bevölferung handelt. Wie bie gejamte menidflide (antwidflung, fo jobreitet auch bie Redtsentwidflung nidjt iprungmeije, jondern jđrittweije und jtufenweife porwärts. Ber fid vermeffen mollte, biejes natürlid)e Gejes nidyt ju beađten, würbe ben ficheren BoDen unter ben Füfen verlieren und ein Strafredht idjaffen, das vielleidht überhaupt nidht, oder nur für eine ferne 3ufunft, jebenfalls aber nidht für bie Begenwart, für bie er jøaffen foll, braudbbar und möglid wäre. Der Entmuri hält es baher für jeine riditig

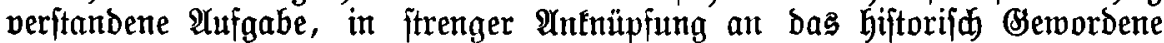
das allgemeine Strafredt auj biejenige Stufe zut heben, bie nadt) ben jegt herrichenden überzeugungen als bie nähjit höhere anzujehen ijt. Er mill bewustermeije auf Den zweifellyaften Borzug blendenber, aber nidjt probe=

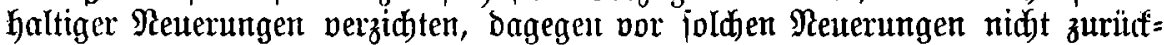

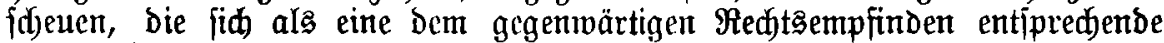

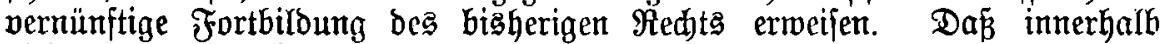

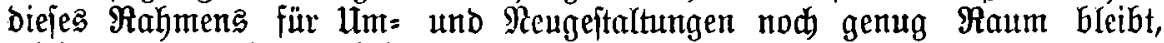
wird aus bem (5ntwurf jelbłt und feiner Begründung hervorgehen.

Der Entwurf vertritt nidft Den Standpunft einer bejtimmten wijןen=

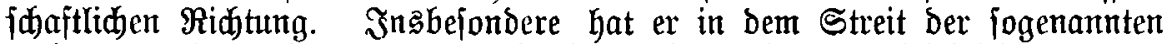

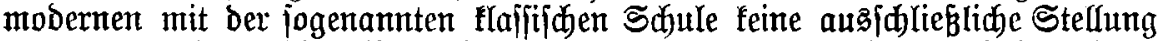
genommen, benn bies ift nidht bie Uufgabe bes praftifanen Gejeggebers. Die ausjabließ̧liche Befolgung einer bejtimmten Theorie führt bei frrenger

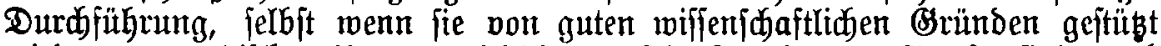
wird, zu praftifiden Unzuträgliafeiten. Die Zwedfe ber Strafe find aud nidjt lebiglid) aus einem (Sejiditspunft zu exfajfen, fondern wie fajt alle menjdylidjen Einridbtungen aus mehreren; Bergeltung, Bejferung, Sdyus ber Sejellidhajt, Beneral= und Spezialpräbention liegen zufammen in ihl

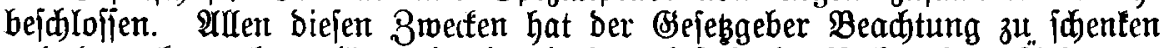

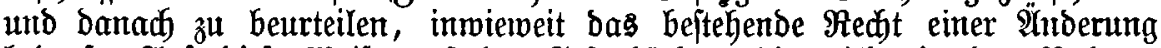

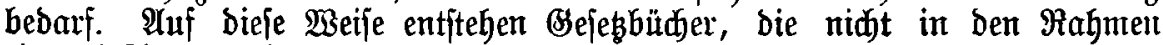
einer beftimmten jtrafrechtlidben Sdulule pajien, und man wirb ifnen aus diejem Umftand feinten Bormurf madjen fönnen. Die anderen modernen (Bejebesarbeiten find ebenfalls feinen anderen $\mathfrak{B e g}$ gegangen ${ }^{1}$ ), und ifn

1) Diez fanm felbft von Dem Rorwegijđen Strafgefełbud gefagt werben, ob=

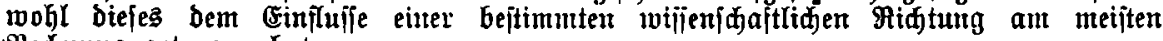
Mednung getragen hat. 


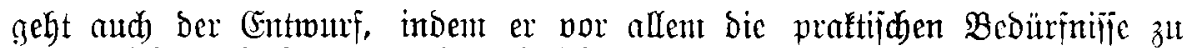

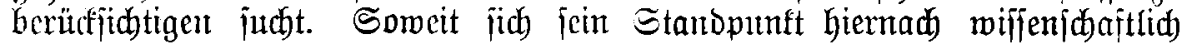

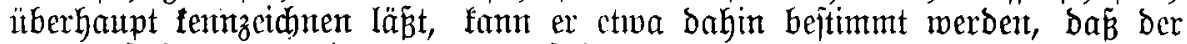

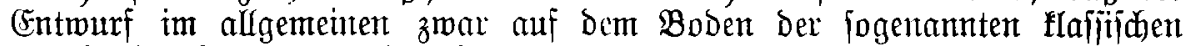

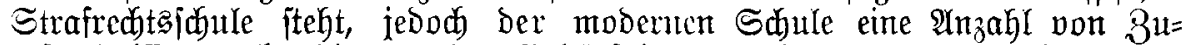

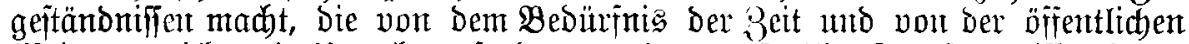

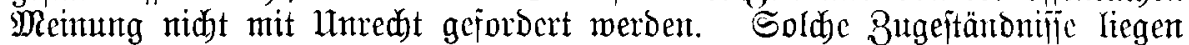
bejonders in den Borjchlägen, die auj (sinführuntg Der jogentannten "be=

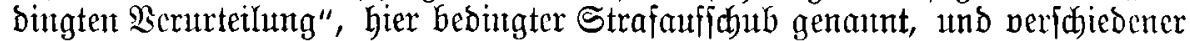

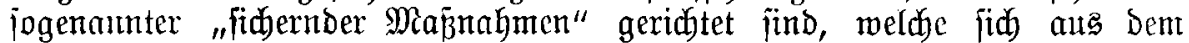
Bergeltungaggedanfen allein nidht redttiertigen laîfen, jondern auf Prävention abjiclen. g̈hnlidb handeln aud anbere neue Bejebgebungen, bejonder's bie Der Edyweiz und Rorwegens. Endlich haben biejen Standpuntt aud nam= hajte Giclehrte aus beiben Lagern als eincn mögliden und ridftigen be=

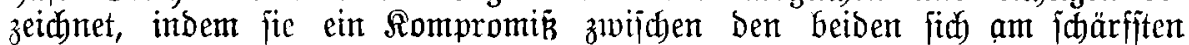

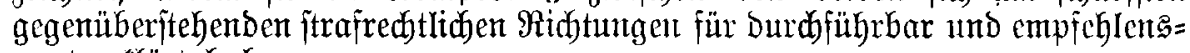
wert erelärt haben.

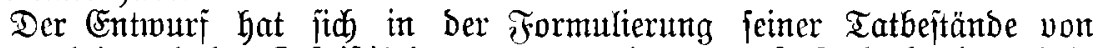
Der Starrbeit und Der Rafuiftif Des gegenmärtigen Strafgejebbudfs in erbeb= lidfem Miake losgejagt, jeine Bejtimmungen elajtifher geftaltet unto es vor=

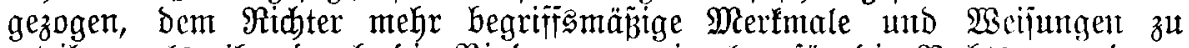

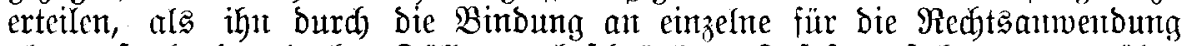

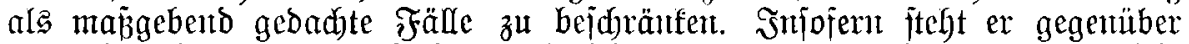

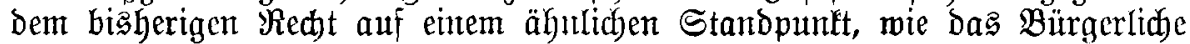

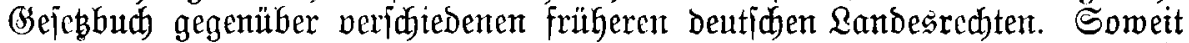
wie biejes burfte er jebod) nidyt gehen. Dem bie Ratur bes Straf=

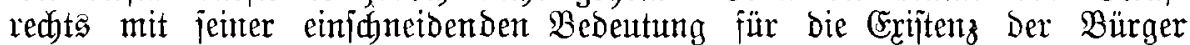

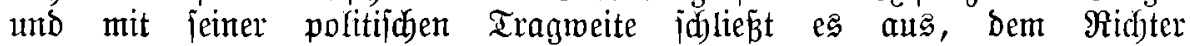

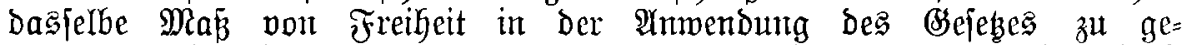

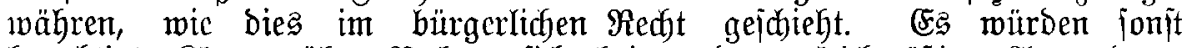

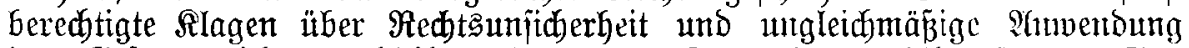
ber Bsejege nidjt ausbreiben Ë̈ntuen. Der alte wohlbegrüntocte Sağ nulla poena sine lege, ber unter andcrem audd ein Redht ber Biijen=

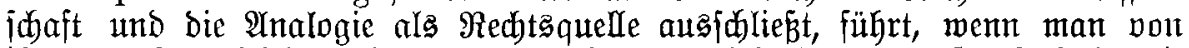

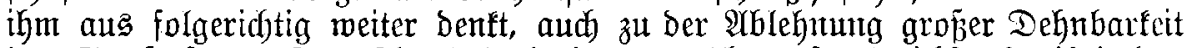
ber Strafgejese. Das Jobeal berjentigen, welde auf möglidjte Freiheit bes ridfterliden Ermeffens hinarbeiten, müste Der Saz jein, Der das Strafred)t

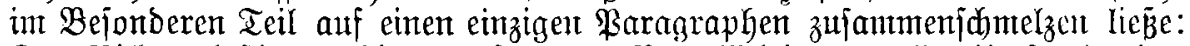

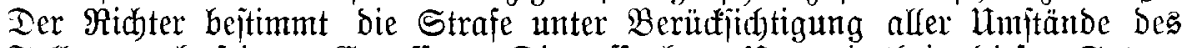
Falles nadb feinem Ermelfen. Die Dffenbare Ungereimtheit biefes Sakes,

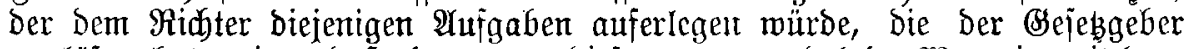

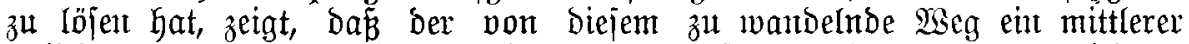

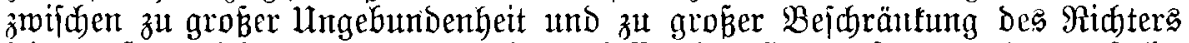

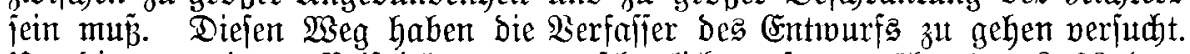

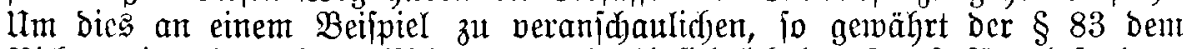

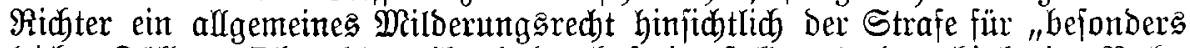

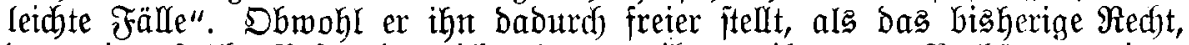
bas eine folde Befugnis nidyt fernt, gibt er ifgnt jur Berfütung eines faj)ranfenlojen und unangemeffenen Bebraud) biefer Befugnis zugleich eine

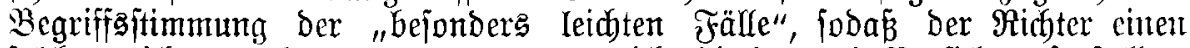
foldyen nidft anmehmen fanm, wenn er nidyt bie bort mit Borfidyt aufgeftellten 


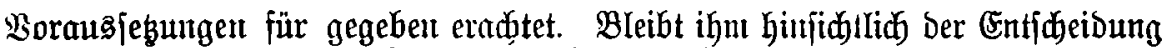
diejes legten \$unftes nod, genug Spielraum für jeit (5rmelien, fo ift biejes ১od fein unbejdränftes, jondein ein an bas Borhandenjein gewifjer tatjäd

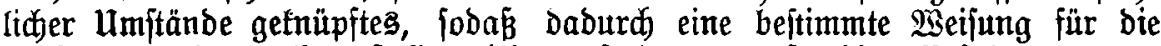
Recht:anwendung hergejtellt wirb, auf beren verftändige Befolgung man im allgemeinen vertrauen lann.

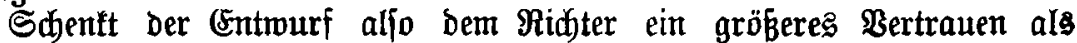

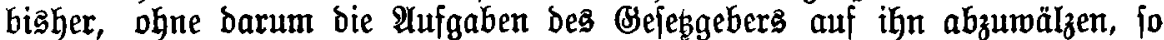
folgt er lebiglid, bem (Bange Der Entwidłelung Der Bejesgebung im allgemeinen. Er erfüllt eime fajt einjtimmige Forberung Der $\mathfrak{B}$ iffenjfdaft, benn, wie nidht

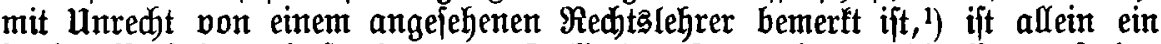
joldhes Berfahren imftande, "an Stelle bes Formalismus bie Bernunft ber Sadje zu retten." (5r folgt audch bem Borgang anderer neuer Bejebgebungen. Die in ber (Segenwart in ber Diffentlidfleit umgehenden, meift politifä gefärbten Rlagen über mangelndes Bertrauen ber Bepölferung zu Den Strafgeridten

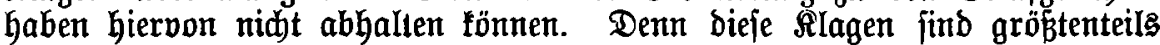
unbegrübet. Die Bepolferung hat weber einen triftigen Grumb zu einem

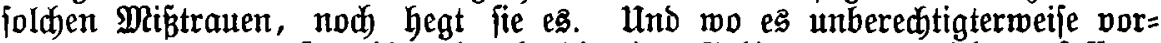

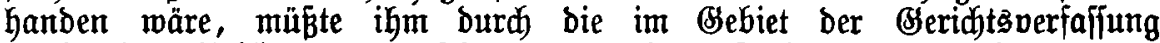

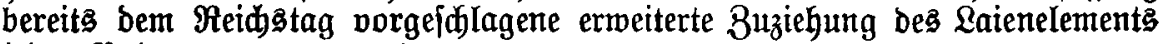
jeber Boben entzogen werben.

Mit biefer allgemeinen Stellungnahme hängt es zujammen, då̉ Der (Entwurf in etwas weiterem Umfang, als bisher, aud $\mathfrak{B o r j d}$ riften gibt, bie

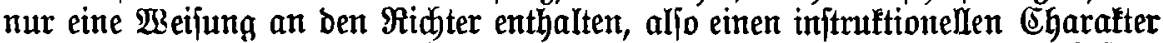

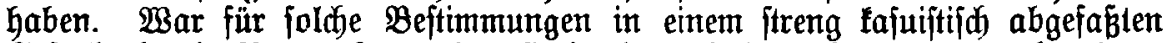

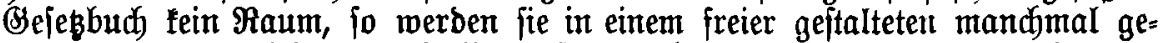

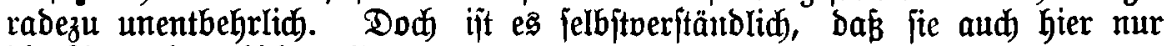
die $\mathfrak{A}$ usnahme billoen fönnett.

Auf Einzelheiten einzugehen, ift hier noch nidht ber Drt. Mur folgendes mag zur allgemeinen §ennzeichnumg vormeg nod bemertt werben:

Die viel angefodtene Dreiteilung ber ftrafbaren bandlungen in $\mathfrak{B e r}=$ brechen, Bergehen und litbertretungen ift bejtehen geblieben, ba nidyt an= erfannt werbent fonnte, daß̧ eine anbere Einteilung befjer wäre, und namentlich bie in einigen anberen Staaten angenommene Bweiteilung in Berbredjen und Übertretungen als für unjere Berhältnifje nidht pajjend angejehen wurbe.

$\mathfrak{A} \mathfrak{n}$ Dem biahberigen Strafeninjtem ift grunbfäblid fejtgehalten, wenn aud) mit nicht unerheblidłen Beränberungen. Die $\mathfrak{B e r f a j f e r ~ w a r e n ~ D e r ~} \mathfrak{A} \mathfrak{n}=$

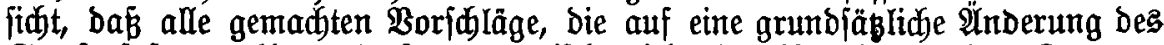
Strafeninjtems hinauslaufen, praftijdy nidht Durdffübrbar finb. Dagegen

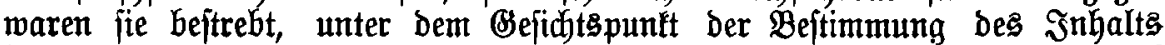

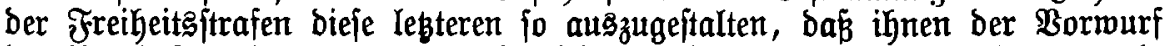

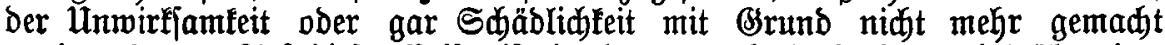
merben fann. YUf bieje Beije ijt in dem Strafgefesbud jo viel über ben Bollzug jener Strafen bejtimmt, baßj allenfalls ein bejonbereả Strafoollzugas=

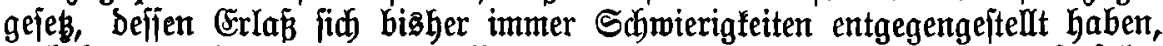
entbehrt und das Weitere Der Berwaltung überlafien merben lann. Snfolge ber $\mathfrak{B e r b e f f e r u n g ~ b e s ~ S t r a f i n h a l t s ~ f a n n ~ a u d ~ e i n e ~ ( E r h o ̈ h u n g ~ b e r ~ M i n d e f t m a ß ̧ e ~}$ ber Freiheitştrafen unterbleiben. Rurzzeitige Freiheitsftrafen find, jo fehr in

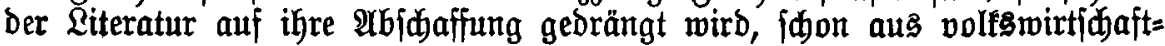

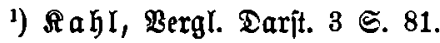




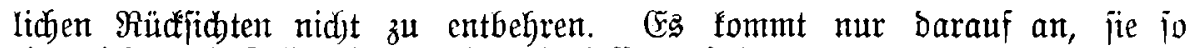
einzuridyten, bå̉ fie bie Strafzmedte belier erfüllen.

Da $\mathfrak{\beta}$ bie jogenannte "bebingte Berurteilung" vorgefdilagen wirb, ij̄t

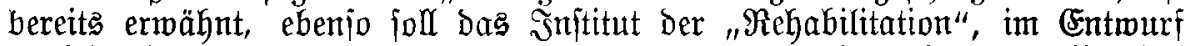
"Wiebereinfebung" genannt, eingeführt werben. Die Sduldehre ift nidjt

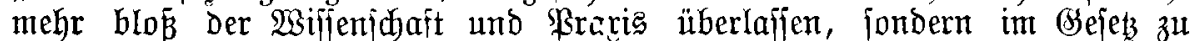
regeln verjudjt worben. Ferner gibt Der (Entwuri eingebende Borjdriften über die Strafbemeliung, von benen bie midhtigften bie Einführung eines allgemeinen Milderungsredts bes Ridjters in "bejonders leidten Fällen", einer allgemeinen Beftimmung gegen Den $\Re$ Audfall und bie Begriffbeftimmung Der "bejonders jadweren Fälle" find, mit Denen Der Entwurf im Bejonderen Teil in bebeutendem umfang ftatt ober neben ben bisher fajuififif formu= lierten eridhwerenden Unftänden arbeitet. Die anertennung einer bejonderen milberen Bejtrafung ber "gemindert" Burednungäähigen, eine neue $\mathfrak{B e}=$ handlung ber jugendlidyen Berjonen (Darunter bic Serlegung ber Grenze Der abjoluten Strafunmündigfeit auf Dag vollendete 14. Lebengjabr), endlid) Die Cinfügung einer Reihe jogenaunter "fichernber Mażnahmen", barutter eine bebeutende Ausdehnung ber Intwendutg bes arbeitghanjes, aber aud) bie ridyterlidye Finmeijung in Irren= und in Irinfer=\$eilanjtalten find

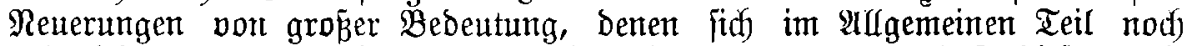

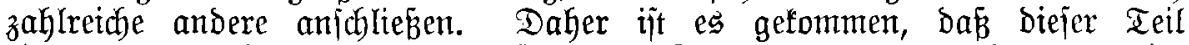
einen um 20 Baragraphen größzeren Hmfang angenommen gat, als im geltenden Bejeb. Dajür find biejem gegenüber im Bejonderen Teil 80 Para=

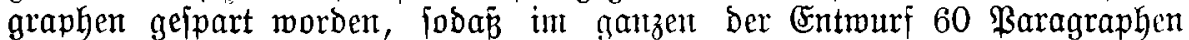
weniger enthält, als bas Strafgeiebbud. Diefe Eriparnis ift wentqer burd)

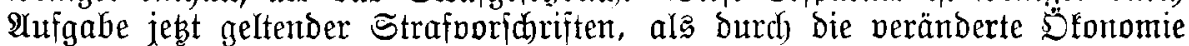

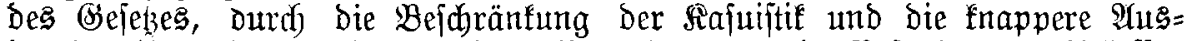

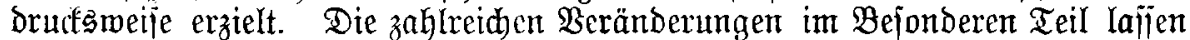
jidh hier weber aufzählen nod) fentzeiduten. Nur bas eine jei bemerft,

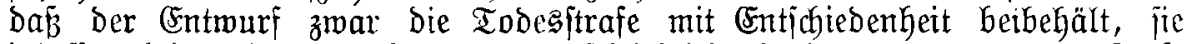
indejien beim Morbe nidht mebr augid)liejlich, jondern nur als Regelijtrafe

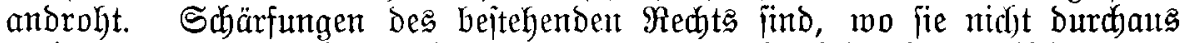
nötig waren, tunlidbjt vermieden, Dagenen mandhe bebeutiame Milberungen eingeführt. Die Strafrahmen find meift elajtijder gejtaltet, um eine größ̈ere snbivibualifierung zu ermöglichen. Bon erhöhten Mindeftitrafen ift aus bent=

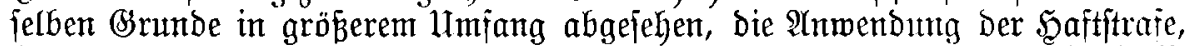
befonders aud bei jogenanten politijathen Deliften, unb ber Gelditrafe ijt bebeutend erweitert, bie Şödhitmaß̉e ber lebteren bagegen find, bem gejunfenen (Seldwert und Den jonit veränderten wirtiḑaftlichen Berhältnifien entipredjent, öfters nidbt unbeträdhtlid erhöht. Die Sinteilung i perändert, einzelne

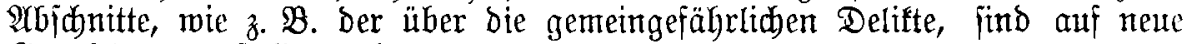
Grundlagen geitellt morben.

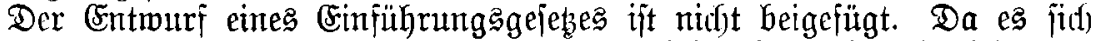
nur un einten Borentmurf handelt, der wahridseinlidi nodf zahlreidje und vielleidht tiefgreifende S̈nderungen erfahren wirb, wäre bies eine untnötige Diühe gewejen. Sin Ginfübrungggejes wirb augzuarbeiten jein, fobald ein zur Borlegung an die gejebgebenden Rörperichaften bejtimmter Entmuri feitgejtellt jein wird.

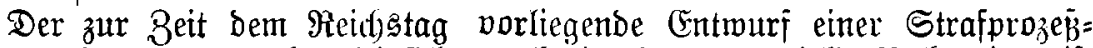

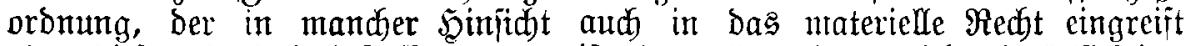
vber biefes bod beeinfluffen fann, ijt in allgemeinen midgt berülfichtigt, 


\section{- XIII -}

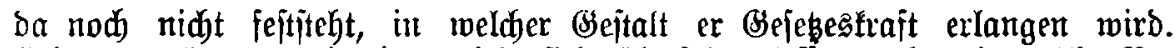
(Erjt wenn lebteres eintritt, wirb jid ïberichen laifen, ob und weldhe $\mathfrak{B} e=$ jitimmungen biejes Entmurfs geändert weroen müfjen. Nur mit ber Ein= führung Der Berufung haben Defifen Berfajijer als mit einer fictjeren Taljache

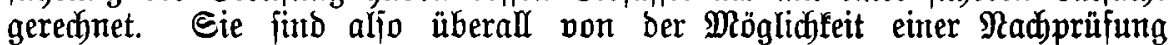
audh ber Tat= und Sdfulbirage in einer oberen İnitanz auşgegangen.

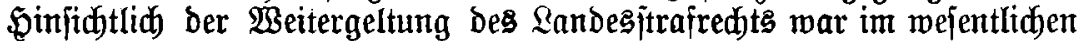
an bem biaherigen $\Re$ edyt feftzulfalten. Die erforberlidyen Beftimmungen werben im Einfühtungşgefeel zu treffen jeir. 
